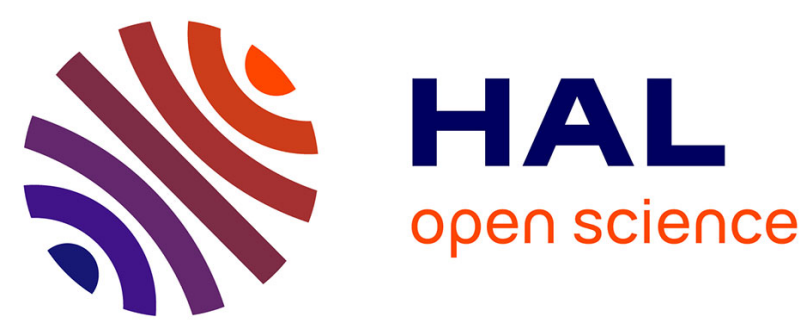

\title{
A self-contained progress variable space solution method for thermochemical variables and flame speed in freely-propagating premixed flamelets
}

\author{
A. Scholtissek, Pascale Domingo, L. Vervisch, C. Hasse
}

\section{- To cite this version:}

A. Scholtissek, Pascale Domingo, L. Vervisch, C. Hasse. A self-contained progress variable space solution method for thermochemical variables and flame speed in freely-propagating premixed flamelets. Proceedings of the Combustion Institute, 2019, 37 (2), pp.1529-1536. 10.1016/j.proci.2018.06.168 . hal-02007788

\section{HAL Id: hal-02007788}

\section{https://hal-normandie-univ.archives-ouvertes.fr/hal-02007788}

Submitted on 4 Dec 2020

HAL is a multi-disciplinary open access archive for the deposit and dissemination of scientific research documents, whether they are published or not. The documents may come from teaching and research institutions in France or abroad, or from public or private research centers.
L'archive ouverte pluridisciplinaire HAL, est destinée au dépôt et à la diffusion de documents scientifiques de niveau recherche, publiés ou non, émanant des établissements d'enseignement et de recherche français ou étrangers, des laboratoires publics ou privés. 


\title{
A self-contained progress variable space solution method for thermochemical variables and flame speed in freely-propagating premixed flamelets
}

\author{
A. Scholtissek ${ }^{\mathrm{a}, *}$, P. Domingo ${ }^{\mathrm{b}}$, L. Vervisch ${ }^{\mathrm{b}}$, C. Hasse $^{\mathrm{a}}$ \\ ${ }^{a}$ Institute for Simulation of reactive Thermo-Fluid Systems, TU Darmstadt, Otto-Berndt-Straße 2, Darmstadt 64287, Germany \\ ${ }^{b}$ CORIA CNRS, Normandie Université, INSA de Rouen, Technopôle du Madrillet, BP 8, 76801 Saint-Étienne-du-Rouvray, France
}

\begin{abstract}
Flamelet models for premixed combustion, which are based on equations formulated and solved in progress variable space, have been proposed in the past, but have not been adopted for chemistry reduction methods. This is due to one limitation of these models: they need a closure for both the magnitude and the shape of the gradient (or scalar dissipation rate) of the progress variable, which is essential for an accurate prediction of the flame displacement speed. So far, solution methods for the aforementioned models require gradient information as an input, which is either modelled and non-generic, or extracted from a previous physical space flame solution for the analogous problem. The objective of this work is to provide a self-contained solution method for freely-propagating premixed flamelets in progress variable space, by solving an additional flamelet equation for the gradient of the progress variable. With this, the novel method provides both magnitude and shape of the gradient. Studying hydrogen-air and methane-air configurations, it is demonstrated that an accurate prediction of the laminar flame speed without the necessity for further input parameters can be obtained.
\end{abstract}

Keywords: premixed flamelet, progress variable space, gradient equation, self-contained, laminar flame speed

\footnotetext{
${ }^{*}$ Corresponding author:

Email address: scholtissek@stfs.tu-darmstadt.de (A. Scholtissek) 


\section{Introduction}

Flamelet [1] and flamelet-based models, such as Flamelet-Generated Manifolds (FGM) [2], Flame Prolongation of ILDM (FPI) [3] or the Reaction/Diffusion Manifold (REDIM) concept [4], have been successfully used as reduction methods for CFD-simulations of chemically reacting flows. Thermochemical states of generic flame configurations are computed a-priori and stored in flamelet tables as a function of few control variables, such as mixture fraction and/or progress variable. In comparison to full chemistry solutions, these methods exhibit a dramatically reduced computational cost by solving only for the flow and the controlling variables, while the remaining thermochemical state is obtained from the flamelet table.

Among flamelet models, where equations are formulated and solved in either mixture fraction space (non-premixed combustion) or progress variable space (premixed combustion), only the former have been extensively used and extended for a wide range of conditions and applications. Few works concentrate on premixed flamelet models in composition space [57], although these models show great potential for the aforementioned reduction methods. This is supported by the many successful applications with their physical space counterpart, the FGM method [2], where one-dimensional flamelet equations are formulated and solved in a flame-adapted coordinate system. There is one major reason why progress variable space flamelet models for premixed combustion have not been widely used: they need a closure for both the magnitude and the shape of the gradient (or scalar dissipation rate) of the progress variable, which is essential for an accurate prediction of the flame displacement speed. In [5] and [6] premixed flamelet equations have been solved, but profiles for the scalar gradient were input variables and had to be modelled or obtained from physical space solutions. In [7] premixed flamelet equations were analyzed and their validity was also demonstrated for turbulent premixed flames based on direct numerical simulations (DNS). Furthermore, the gradient (or scalar dissipation rate) of the progress variable is a central parameter in most modeling approaches for turbulent premixed flames [8] since it is linked to the fundamental quantities in turbulent combustion [9].

It is the objective of this work to show how a closure for the scalar gradient of the progress variable can be obtained for unstrained premixed flamelets. Therefore, an additional flamelet equation is solved for the gradient, resulting in a self-contained model which also allows the laminar flame speed to be computed in the progress variable space. Being the counterpart to the freely-propagating flame in physical space, the model presented here covers the most important canonical flame configuration for premixed combustion.

\section{Self-contained Flamelet Formulation}

A freely-propagating premixed flame can be described by the following equation set [10]

$$
\begin{aligned}
\frac{\partial \rho u}{\partial x} & =0 \\
\rho c_{p} u \frac{\partial T}{\partial x} & =\frac{\partial}{\partial x}\left(\lambda \frac{\partial T}{\partial x}\right)-\rho \sum_{k}^{N_{s}} c_{p, k} Y_{k} V_{k} \frac{\partial T}{\partial x}+\dot{\omega}_{T}, \\
\rho u \frac{\partial Y_{i}}{\partial x} & =-\frac{\partial}{\partial x}\left(\rho Y_{i} V_{i}\right)+\dot{\omega}_{i},
\end{aligned}
$$

where $x$ is the spatial coordinate of the one-dimensional physical space, $\rho$ is the density, $u$ the flow velocity, $c_{p}$ the specific heat capacity, $T$ the temperature and $\lambda$ the thermal conductivity. The quantities $Y_{i}$ and $V_{i}$ denote the mass fraction and the diffusion velocity of species $i$, respectively, and the chemical source terms for temperature and species are represented by $\dot{\omega}_{T}$ and $\dot{\omega}_{i}$. The mass flux

$$
\rho u=\rho_{0} s_{l},
$$

is an eigenvalue for the above equation system and is directly linked to the laminar flame speed $s_{l}$ ( $\rho_{0}$ denotes the density of the unburnt gases).

The progress variable $Y_{c}$ is introduced as $Y_{c}=$ $\sum_{i} \alpha_{i} Y_{i}$, where $\alpha_{i}$ is a species-specific weighting factor. An analogous summation of the species equations (Eq. (3)) yields a balance equation for the progress variable:

$$
\rho u \frac{\partial Y_{c}}{\partial x}=-\frac{\partial}{\partial x}\left(\rho Y_{c} V_{c}\right)+\dot{\omega}_{c},
$$

where the diffusive flux of the progress variable is defined as $\rho Y_{c} V_{c}=\rho \sum_{i} \alpha_{i} Y_{i} V_{i}$. Introducing the gradient of the progress variable $g_{c}=\partial Y_{c} / \partial x$ and using Eqs. (4) and 5, an expression for the laminar flame speed is obtained

$$
s_{l}=\frac{1}{\rho_{0} g_{c}}\left(-\frac{\partial}{\partial x}\left(\rho Y_{c} V_{c}\right)+\dot{\omega}_{c}\right) .
$$

\subsection{Transformation Rules}

In the following sections, Eqs. (2), (3) and (5) are transformed and the progress variable $Y_{c}$ is chosen as the control variable. The transformation rule from the physical space coordinate system $x$ to the flame-adapted progress variable space $Y_{c}$ reads

$$
\frac{\partial T}{\partial x}=\frac{\partial Y_{c}}{\partial x} \frac{\partial T}{\partial Y_{c}},
$$


which is applied analogously to other scalar quantities.

\subsection{Flamelet Equations for Equal Diffusivities}

Assuming equal diffusivities for species and temperature, or equivalently unity Lewis numbers, the species diffusion velocity is expressed as

$$
V_{i}=-\frac{D}{Y_{i}} \frac{\partial Y_{i}}{\partial x}
$$

where the diffusivity is defined as $D=\lambda /\left(\rho c_{p}\right)$. Applying the transformation rules in Eq. (7) to Eqs. (2) and (3) (detailed derivations are found in the supplementary material) yields the premixed flamelet equations for temperature and species

$$
\begin{aligned}
& \rho D g_{c}^{2} \frac{\partial^{2} T}{\partial Y_{c}^{2}}-\dot{\omega}_{c} \frac{\partial T}{\partial Y_{c}}+\frac{\dot{\omega}_{T}}{c_{p}}+\frac{\rho D g_{c}^{2}}{c_{p}} \frac{\partial c_{p}}{\partial Y_{c}} \frac{\partial T}{\partial Y_{c}} \\
& +\rho D g_{c}^{2} \sum_{k} \frac{c_{p, k}}{c_{p}} \frac{\partial Y_{k}}{\partial Y_{c}} \frac{\partial T}{\partial Y_{c}}=0 \\
& \rho D g_{c}^{2} \frac{\partial^{2} Y_{i}}{\partial Y_{c}^{2}}-\dot{\omega}_{c} \frac{\partial Y_{i}}{\partial Y_{c}}+\dot{\omega}_{i}=0 .
\end{aligned}
$$

As outlined earlier, an additional flamelet equation is solved for $g_{c}$, which is obtained by differentiating Eq. (5)

$$
\frac{\partial}{\partial x}\left[u \frac{\partial Y_{c}}{\partial x}=-\frac{1}{\rho} \frac{\partial}{\partial x}\left(\rho Y_{c} V_{c}\right)+\frac{\dot{\omega}_{c}}{\rho}\right] .
$$

Applying the transformation rules to this expression yields

$$
\begin{aligned}
& a g_{c}+\frac{\dot{\omega}_{c}}{\rho} \frac{\partial g_{c}}{\partial Y_{c}}=\frac{g_{c}^{2}}{\rho} \frac{\partial^{2}}{\partial Y_{c}^{2}}\left(\rho D g_{c}\right) \\
& -\frac{g_{c}^{2}}{\rho^{2}} \frac{\partial \rho}{\partial Y_{c}} \frac{\partial}{\partial Y_{c}}\left(\rho D g_{c}\right)+g_{c} \frac{\partial}{\partial Y_{c}}\left(\frac{\dot{\omega}}{\rho}\right) .
\end{aligned}
$$

where $a$ is the strain rate, defined as [11, 12]

$$
a=-\frac{\partial u}{\partial x}
$$

Using Eq. (1), $a$ can be related to the eigenvalue of the flame

$$
\frac{\partial \rho u}{\partial x}=0=-\rho a+\frac{\rho_{0} s_{l}}{\rho} \frac{\partial \rho}{\partial x} .
$$

Applying the transformation rule in Eq. (7) and using Eq. (6), the strain rate equals

$$
a=-\frac{g_{c} \rho_{0} s_{l}}{\rho^{2}} \frac{\partial \rho}{\partial Y_{c}}=-\frac{1}{\rho^{2}} \frac{\partial \rho}{\partial Y_{c}}\left(-\frac{\partial}{\partial x}\left(\rho Y_{c} V_{c}\right)+\dot{\omega}_{c}\right) .
$$

This shows that $a$ is non-zero also for an unstrained premixed flame, which is due to thermal expansion since it is a function of the density gradient. Introducing Eq. (15) into Eq. (12) yields

$$
g_{c}^{2} \frac{\partial^{2}}{\partial Y_{c}^{2}}\left(\rho D g_{c}\right)-\dot{\omega}_{c} \frac{\partial g_{c}}{\partial Y_{c}}+g_{c} \frac{\partial \dot{\omega}_{c}}{\partial Y_{c}}=0
$$

Considering their origin in the transformation, the terms are interpreted as (left to right): diffusion, $Y_{c^{-}}$ drift (transformed convective term) and chemical source term. Equations (9), (10) and (16) represent a closed equation system which can be solved in progress variable space.

\subsection{Flamelet Equations with Differential Diffusion}

In this section, the premixed flamelet equations are generalized to capture also differential diffusion effects. Keeping in mind that the species diffusion velocity $V_{i}$ generally contains gradient information, it can be written as

$$
\rho Y_{i} V_{i}=\rho Y_{i} \tilde{V}_{i} \frac{\partial Y_{c}}{\partial x}
$$

where gradients have been transformed according to Eq. (7) and $\tilde{V}_{i}$ represents a diffusion velocity in progress variable space. Instead of gradients with respect to physical space coordinates, $\tilde{V}_{i}$ contains gradients with respect to the conditioning variable $Y_{c}$ and can therefore be computed from standard transport libraries on a $Y_{c^{-}}$ grid. Besides unity Lewis number diffusion, a mixtureaveraged diffusion model [13] with a correction velocity [14] for mass conservation is used in this work. In principle the approach allows arbitrary diffusion models to be transferred to progress variable space.

The diffusive flux of the progress variable is defined as

$$
Y_{c} V_{c}=\frac{\partial Y_{c}}{\partial x} Y_{c} \tilde{V}_{c}=\frac{\partial Y_{c}}{\partial x} \sum_{i} \alpha_{i} Y_{i} \tilde{V}_{i}
$$

which also allows a Lewis number to be defined for the progress variable $\mathrm{Le}_{c}=-\lambda /\left(\rho c_{p} Y_{c} \tilde{V}_{c}\right)$. This information is essential for evaluating the diffusive flux of $Y_{c}$, when using the premixed flamelet model in CFDsimulations. In this case, it was possible to store either $Y_{c} \tilde{V}_{c}$ or $\mathrm{Le}_{c}$ as a function of $Y_{c}$ in a flamelet table.

Using Eq. (17) instead of Eq. (8) in the flamelet transformation, the premixed flamelet equations for $T, Y_{i}$ and 
$g_{c}$ read in generalized form

$$
\begin{aligned}
& \frac{g_{c}}{c_{p}} \frac{\partial}{\partial Y_{c}}\left(g_{c} \lambda \frac{\partial T}{\partial Y_{c}}\right)+g_{c} \frac{\partial}{\partial Y_{c}}\left(g_{c} \rho Y_{c} \tilde{V}_{c}\right) \frac{\partial T}{\partial Y_{c}} \\
& +g_{c}^{2} \sum_{k} \frac{c_{p, k}}{c_{p}} \rho Y_{k} \tilde{V}_{k} \frac{\partial T}{\partial Y_{c}}-\dot{\omega}_{c} \frac{\partial T}{\partial Y_{c}}+\frac{\dot{\omega}_{T}}{c_{p}}=0, \\
& -g_{c} \frac{\partial}{\partial Y_{c}}\left(g_{c} \rho Y_{i} \tilde{V}_{i}\right)+g_{c} \frac{\partial}{\partial Y_{c}}\left(g_{c} \rho Y_{c} \tilde{V}_{c}\right) \frac{\partial Y_{i}}{\partial Y_{c}} \\
& -\dot{\omega}_{c} \frac{\partial Y_{i}}{\partial Y_{c}}+\dot{\omega}_{i}=0, \\
& -g_{c}^{2} \frac{\partial^{2}}{\partial Y_{c}^{2}}\left(g_{c} \rho Y_{c} \tilde{V}_{c}\right)-\dot{\omega}_{c} \frac{\partial g_{c}}{\partial Y_{c}}+g_{c} \frac{\partial \dot{\omega}_{c}}{\partial Y_{c}}=0 .
\end{aligned}
$$

The consistency of these equations to the ones derived in previous works [5-7] is discussed in the supplementary material.

\subsection{Boundary Conditions}

The premixed flamelet equations are subject to the following boundary conditions

$$
\begin{array}{ll}
Y_{c}=Y_{c, \text { min }}: & T=T_{0}, Y_{i}=Y_{i, 0}, g_{c}=0, \\
Y_{c}=Y_{c, \text { eq }}: & T=T_{\mathrm{eq}}, Y_{i}=Y_{i, \mathrm{eq}}, g_{c}=0,
\end{array}
$$

where the subscript 0 denotes the thermochemical state of the fresh mixture and eq marks the chemical equilibrium.

\subsection{Numerical Methods}

The premixed flamelet equations presented in the previous sections are solved with a hybrid Newton method [15] in a multi-grid approach with an in-house $\mathrm{C}++$ flame solver [16]. After the equations are solved on a coarse grid, the grid is adaptively refined until a userspecified target. For the evaluation of diffusion coefficients and chemical source terms, the EGlib [17, 18] and an in-house chemistry library are used, respectively. The GRI-Mech 3.0 [19] is applied for methaneair flames and the mechanism by Varga et al. [20] is applied for hydrogen-air flames.

\section{Results and Discussion}

In this section, general characteristics of the premixed flamelet model are discussed first. Thereafter, flamelet results are compared to the corresponding physical space flame configurations for three premixed flame categories:

C1 methane-air flames assuming equal diffusivities,

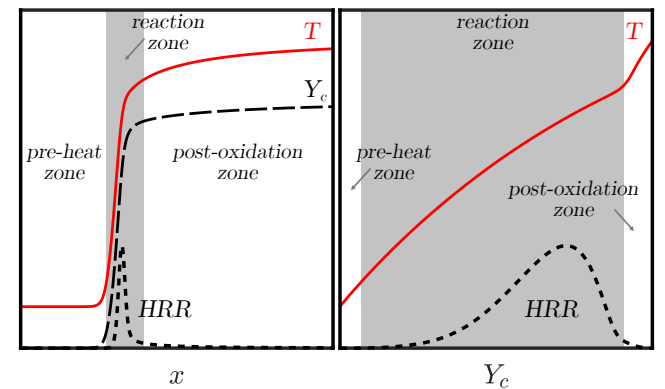

Fig. 1: General characteristics of premixed flamelets - left: Temperature, progress variable and heat release rate (HRR) in physical space. Right: Temperature and heat release rate (HRR) in progress variable space. In comparison to the physical space, the reaction zone is enlarged in progress variable space, while pre-heat zone and postoxidation zone are confined to thin layers at the boundaries.

C2 methane-air flames using a mixture-averaged diffusion model,

C3 hydrogen-air flames using a mixture-averaged diffusion model.

The flamelet solutions are computed by solving Eqs. (19)-(21) as previously outlined and the physical space solutions are obtained by solving Eqs. (1)-(3) with the procedure described in [10]. For all flames the equivalence ratio is varied between 0.8 and 1.2. Finally, the budget of the flamelet equation for the progress variable gradient is analyzed.

\subsection{Premixed Flamelet Characteristics}

In comparison to the representation of premixed flamelets in physical space, the numerical model in progress variable space exhibits different characteristics, as shown in Fig. 1. While the reaction zone is very thin in physical space, it almost spans the whole computational domain in progress variable space. Consequently, gradients can be accurately resolved in the most important part of the flame with fewer points compared to the physical space representation. In contrast to the reaction zone, the flame's pre-heat and post-oxidation zones are confined to thin layers at the boundaries in progress variable space. This poses numerical difficulties for an accurate and robust solution method for the premixed flamelet equations. In the pre-heat zone differential diffusion effects might occur while there is minimal reaction progress. To properly resolve the differential diffusion effects, especially for light species such as $\mathrm{H}_{2}$, the grid must be refined towards the pre-heat zone and the reaction progress variable should contain some of the light species. Otherwise, their profiles might exhibit large gradients in the pre-heat zone, which leads to errors in the laminar flame speed of above $5 \%$ for the 

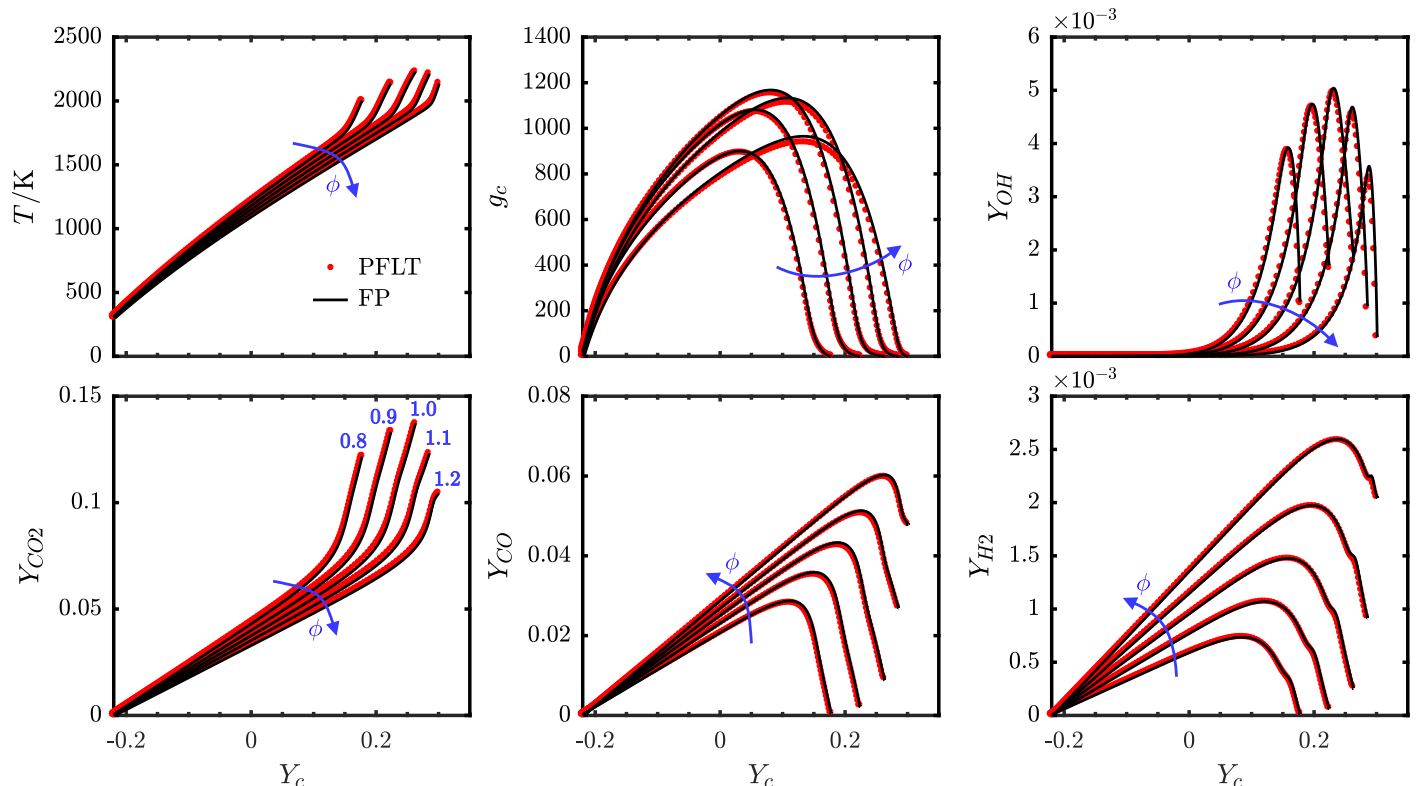

Fig. 2: Comparison of premixed flamelet solutions $(\mathrm{dot})$ to freely-propagating flame solutions (line) assuming equal diffusivities $($ Le $=1)$ for methane-air flames for different equivalence ratios (0.8-1.2, blue). The progress variable is defined as $Y_{c}=Y_{\mathrm{CO}}+Y_{\mathrm{CO}_{2}}+Y_{\mathrm{H}_{2} \mathrm{O}}+10 Y_{\mathrm{H}_{2}}-Y_{\mathrm{O}_{2}}$.

flames studied in this work. In the post-oxidation zone, the gradient of the progress variable becomes small and slow chemical reactions control this region. Asymptotically, as $g_{c} \rightarrow 0$, diffusive terms vanish in the flamelet equations and the equations for the homogeneous reactor in progress variable space are recovered [21]:

$$
\frac{\partial Y_{i}}{\partial Y_{c}}=\frac{\dot{\omega}_{i}}{\dot{\omega}_{c}} \quad \text { and } \quad \frac{\partial T}{\partial Y_{c}}=\frac{1}{c_{p}} \frac{\dot{\omega}_{T}}{\dot{\omega}_{c}} .
$$

The above equation set allows only one boundary condition to be specified. Thus, even if $g_{c}$ is non-zero, but very small, the information about the post-oxidation zone (in the limit an equilibrium boundary condition) shows very little effect on the reaction zone. Alternatively to prescribing the (exact) equilibrium conditions in the post-oxidation zone, it is also possible to define the boundary condition as an open boundary, solving the premixed flamelet equations at this point. With this, the thermochemical state in the post-oxidation zone is a result of the computation as it is similarly the case for freely-propagating flames in physical space which recover close-to-equilibrium conditions at the flow outlet. Albeit this theoretical insight, both methods are interchangeable and for all computations discussed below the boundary conditions as outlined in Sec. 2.4 are used.

\subsection{Temperature, Species and Gradient Profiles}

Figure 2 shows the solutions of the temperature, the gradient $g_{c}$ and four species for the methane-air flames with equal diffusivities (C1). Overall, the premixed flamelet model with equal diffusivities accurately reproduces the physical space solution for all displayed variations of the equivalence ratio. For the cases discussed here, the freely-propagating flame solutions were computed with a multi-grid approach on 1000 points, while the progress variable space model was computed on 200 points (both adaptively refined from approximately 20 starting points). The progress variable is defined by $Y_{c}=Y_{\mathrm{CO}}+Y_{\mathrm{CO}_{2}}+Y_{\mathrm{H}_{2} \mathrm{O}}+10 Y_{\mathrm{H}_{2}}-Y_{\mathrm{O}_{2}}$ for consistency with the computations with mixture-averaged diffusion (C2), which further accounts for differential diffusion. However, for the cases with equal diffusivities, a simpler definition of the progress variable would also yield similar results.

The results for the methane-air flames with non-unity Lewis numbers are displayed in Fig. 3. In accordance with the previous results, the premixed flamelet model accurately reproduces all solution quantities. Although they are very similar to the results from Fig. 2, differences due to differential diffusion are notable in the profiles of $g_{c}, \mathrm{CO}_{2}$ and especially $\mathrm{H}_{2}$. As mentioned earlier, the $\mathrm{H}_{2}$ shows large gradients in the pre-heat zone due to differential diffusion in the pre-heat zone. If $\mathrm{H}_{2}$ was not contained in the definition of $Y_{c}$, it becomes very challenging for the premixed flamelet model to capture this profile accurately (not shown here). From this observation it becomes evident, that the accuracy of the model poses more constraints on the definition of the progress 

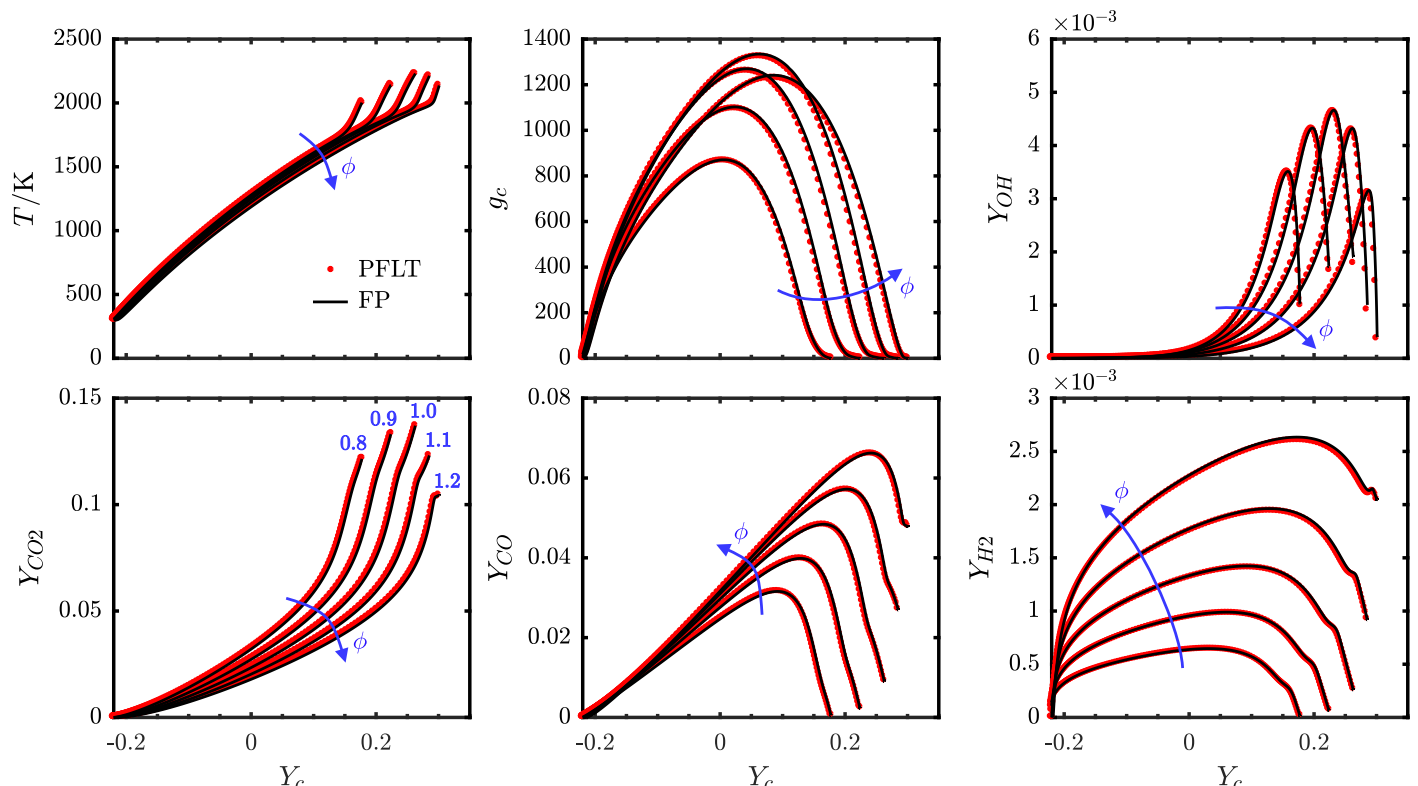

Fig. 3: Comparison of premixed flamelet solutions (dot) to freely-propagating flame solutions (line) using a mixture-averaged diffusion model for methane-air flames for different equivalence ratios (0.8-1.2, blue). The progress variable is defined as $Y_{c}=Y_{\mathrm{CO}}+Y_{\mathrm{CO}_{2}}+Y_{\mathrm{H}_{2} \mathrm{O}}+10 Y_{\mathrm{H}_{2}}-Y_{\mathrm{O}_{2}}$.

variable beyond strict monotonicity.

In Fig. 4 hydrogen-air flames are shown for different equivalence ratios. Although $\mathrm{H}_{2} \mathrm{O}$ is a common choice for the progress variable for these flames, again $\mathrm{H}_{2}$ had to be included in its definition for a sufficient accuracy of the model. As already observed for the methaneair flames, the model correctly reproduces the physical space flame results. This is also true for light radical species such as $\mathrm{H}$, which underlines the fact that the premixed flamelet model correctly captures differential diffusion effects.

The comparison of the freely-propagating flames to the premixed flamelet solutions illustrates, that the closure for the conditional strain rate Eq. (13) is exact and can be used to accurately predict the progress variable gradient $g_{c}$. Inspecting the shape of all gradient profiles (C1-C3) shows, that there does not exist a generic profile for premixed flames, hence $g_{c}$ must be a solution quantity.

\subsection{Comparison of the Laminar Flame Speeds}

In progress variable space, the laminar flame speed $s_{l}$ can be computed by transforming Eq. (6)

$$
s_{l}=\frac{1}{\rho_{0}}\left[-\frac{\partial}{\partial Y_{c}}\left(g_{c} \rho Y_{c} \tilde{V}_{c}\right)+\frac{\dot{\omega}_{c}}{g_{c}}\right] .
$$

In this work, $s_{l}$ is evaluated at the normalized progress variable $c=0.5$. A comparison of $s_{l}$ between the result from the progress variable space model and the physical space solution for all cases $(\mathrm{C} 1-\mathrm{C} 3)$ is displayed in Fig. 5. The premixed flamelet model reproduces $s_{l}$ with less than $2.5 \%$ difference as compared to the physical space computations.

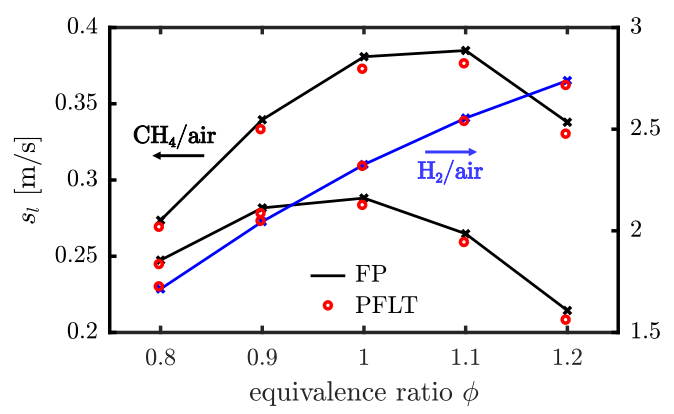

Fig. 5: Laminar flame speeds (C1-C3) for the physical space solution (lines with symbols) and for the premixed flamelet model (red dots).

\subsection{Analysis of the $g_{c}$ Budget}

Figure 6 shows the budget analysis for the $g_{c}$ flamelet equation Eq. (21). It is observed that the diffusive term and the source term are important throughout the whole domain, while the $Y_{c}$-drift term shows major influences only in the region with considerable reaction progress where $c>0.6$. This characteristic coincides with the maximum of the progress variable source term, which is shifted to the right of the computational domain, analogously to the heat release rate in Fig. 1. The budget shows that both terms which contain the chemical 

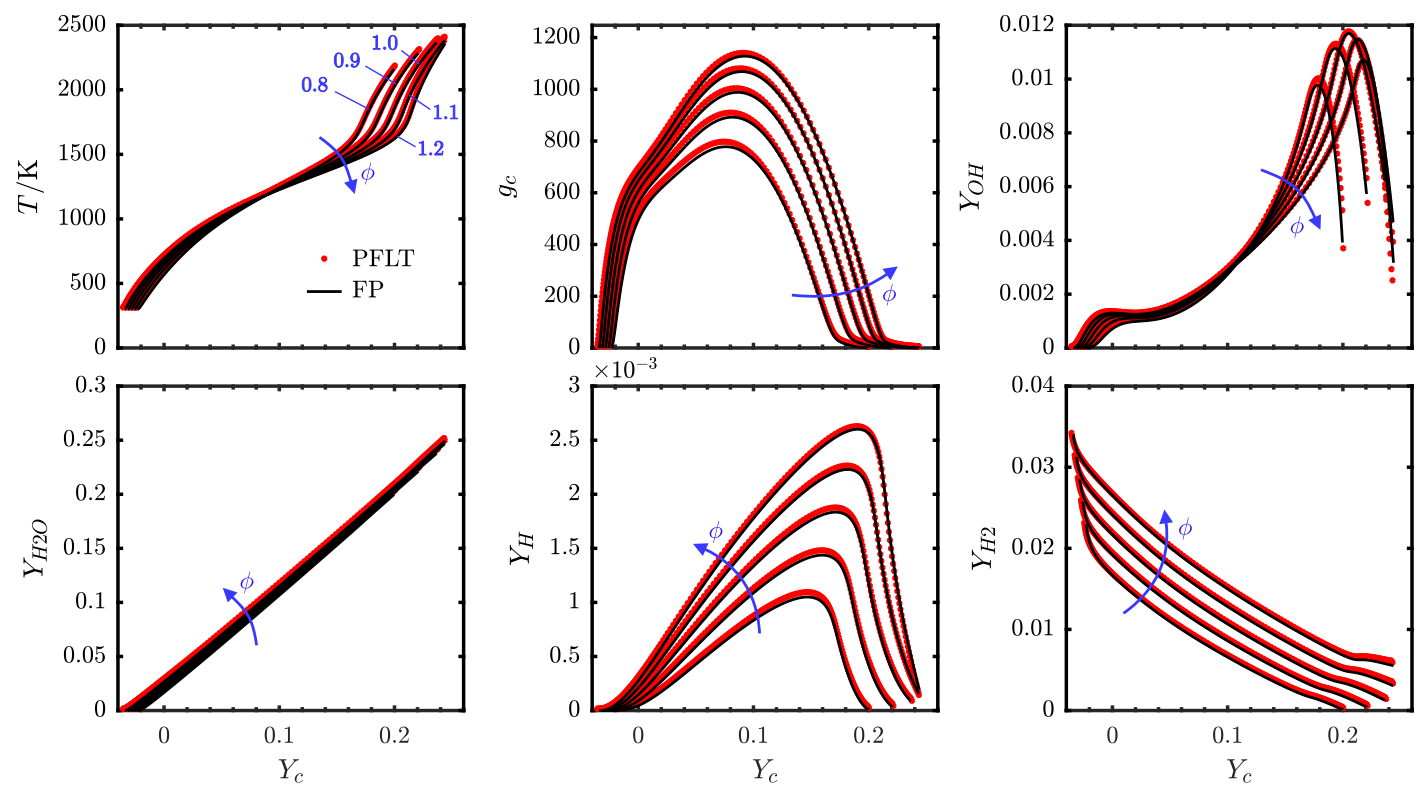

Fig. 4: Comparison of premixed flamelet solutions (dot) to freely-propagating flame solutions (line) using a mixture-averaged diffusion model for hydrogen-air flames for different equivalence ratios $\left(0.8-1.2\right.$, blue). The progress variable is defined as $Y_{c}=Y_{\mathrm{H}_{2}} \mathrm{O}-Y_{\mathrm{H}_{2}}$.

source term of the progress variable play a dominant role. Obviously, these terms would not be present if the control variable was a passive scalar, such as the mixture fraction for non-premixed flames. While nonpremixed flamelets require a forcing term in the form of strain to sustain a steady flame (also evident from the equation for the scalar dissipation rate, see [11]), this is not the case for premixed flamelets. However, it would make sense to also introduce such a term in the premixed flamelet model to extend it to capture influences of imposed strain. A corresponding method in physical space is the strong stretch theory for FGM-models [2].

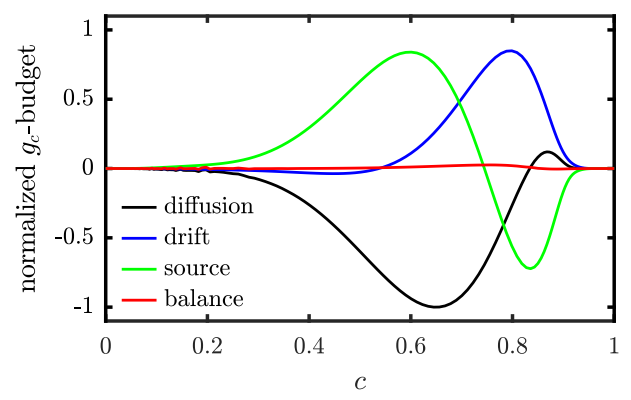

Fig. 6: Normalized budget of Eq. (21) over the normalized progress variable $c$ for a stoichiometric methane-air flame. The balance represents the sum of the three terms.

\section{Summary}

In this work, a self-contained flamelet model for steady unstrained premixed flames is presented. Flamelet equations for temperature, species and the gradient of the progress variable are formulated and solved in progress variable space, which also yields a closure for the laminar flame speed. A generic transformation of the species diffusive flux is introduced, such that it can be computed with standard transport libraries on a $Y_{c}$-grid. With this, the model can capture differential diffusion effects, since common diffusion models may also be used in progress variable space. The premixed flamelet model is then applied to three different flame categories, varying the equivalence ratio: methane-air flames assuming equal diffusivities (C1), methane-air flames using a mixture-averaged diffusion model (C2) and hydrogen-air flames using a mixture-averaged diffusion model (C3). It is shown that for all cases the model accurately captures the profiles of the solution quantities in accordance with the corresponding freelypropagating flame calculations in physical space. Furthermore, the progress variable space solution method can predict the laminar flame speed with differences less than $2.5 \%$ compared the physical space solution, where it is an eigenvalue.

The model presented here complements flamelet modelling approaches for the most important canonical premixed flame configuration. It remains a subject for future work to show how strain and curvature effects 
can be consistently incorporated into a progress variable space flamelet model and to explore how the model may be integrated into flamelet approaches for turbulent flames.

\section{Acknowledgments}

Financial support is kindly acknowledged from the German Research Foundation (DFG) in the Project HA 4367/4-1.

\section{References}

[1] N. Peters, Symp. (Int.) Combust. 21 (1988) 1231-1250.

[2] J. A. van Oijen, A. Donini, R. J. M. Bastiaans, J. H. M. ten Thije Boonkkamp, L. P. H. de Goey, Prog. Energy Combust. Sci. 57 (2016) $30-74$

[3] O. Gicquel, N. Darabiha, D. Thévenin, Proc. Combust. Inst. 28 (2000) $1901-1908$.

[4] V. Bykov, U. Maas, Combust. Theor. Model. 11 (2007) 839862.

[5] P.-D. Nguyen, L. Vervisch, V. Subramanian, P. Domingo, Combust. Flame 157 (1) (2010) $43-61$.

[6] G. Lodier, L. Vervisch, V. Moureau, P. Domingo, Combust. Flame 158 (10) (2011) 2009 - 2016.

[7] B. Savard, G. Blanquart, Combust. Flame 180 (2017) 77-87.

[8] H. Kolla, Scalar dissipation rate based flamelet modelling of turbulent premixed flames, Ph.D. thesis, University of Cambridge, U.K. (2009). doi:10.17863/CAM.13979.

[9] D. Veynante, L. Vervisch, Prog. Energy Combust. Sci. 28 (2002) $193-266$.

[10] R. J. Kee, J. F. Grcar, M. D. Smooke, J. A. Miller, E. Meeks, PREMIX: a fortran program for modeling steady laminar onedimensional premixed flames, Tech. Rep. SAND85-8249, Sandia National Laboratories (1985)

[11] C. Hasse, N. Peters, Proc. Combust. Inst. 30 (2005) 2755-2762.

[12] N. Peters, L. Wang, $5^{\text {th }}$ US Combustion Meeting, San Diego, California, The Combustion Institute, 2007.

[13] C. F. Curtiss, J. O. Hirschfelder, J. Chem. Phys. 17 (1949) 550555.

[14] T. P. Coffee, J. M. Heimerl, Combust. Flame 43 (1981) 273-289.

[15] J. F. Grcar, R. J. Kee, M. D. Smooke, J. A. Miller, Symp. (Int.) Combust. 21 (1988) $1773-1782$.

[16] A. Zschutschke, D. Messig, A. Scholtissek, C. Hasse, online (2017). doi:10.6084/m9.figshare.5119855.v2.

[17] A. Ern, V. Giovangigli, Multicomponent Transport Algorithms, Springer Berlin Heidelberg, 1994.

[18] A. Ern, V. Giovangigli, EGLIB: a General-Purpose Fortran Library for Multicomponent Transport Property Evaluation, Tech. rep., CERMICS (1996).

[19] G. P. Smith, D. M. Golden, M. Frenklach, N. W. Moriarty, B. Eiteneer, M. Goldenberg, C. T. Bowman, R. K. Hanson, S. Song, W. C. G. Jr., V. V. Lissianski, Z. Qin, Gri-mech 3.0, online (1999).

URL http://www.me.berkeley.edu/gri_mech/

[20] T. Varga, T. Nagy, C. Olm, I. Zsély, R. Pálvölgyi, É. Valkó, G. Vincze, M. Cserháti, H. Curran, T. Turányi, Proc. Combust. Inst. 35 (2015) 589-596.

[21] P. Domingo, L. Vervisch, D. Veynante, Combust. Flame 152 (2008) $415-432$. 


\section{List of Figures}

1 General characteristics of premixed flamelets - left: Temperature, progress variable and heat release rate (HRR) in physical space. Right: Temperature and heat release rate (HRR) in progress variable space. In comparison to the physical space, the reaction zone is enlarged in progress variable space, while pre-heat zone and post-oxidation zone are confined to thin layers at the boundaries. . . . . . . .

2 Comparison of premixed flamelet solutions (dot) to freely-propagating flame solutions (line) assuming equal diffusivities $(\mathrm{Le}=1)$ for methane-air flames for different equivalence ratios $(0.8-1.2$, blue). The progress variable is defined as $Y_{c}=Y_{\mathrm{CO}}+Y_{\mathrm{CO}_{2}}+Y_{\mathrm{H}_{2} \mathrm{O}}+10 Y_{\mathrm{H}_{2}}-Y_{\mathrm{O}_{2}} \ldots \ldots \ldots \ldots$

3 Comparison of premixed flamelet solutions (dot) to freely-propagating flame solutions (line) using a mixture-averaged diffusion model for methane-air flames for different equivalence ratios (0.8-1.2, blue). The progress variable is defined as $Y_{c}=Y_{\mathrm{CO}}+Y_{\mathrm{CO}_{2}}+Y_{\mathrm{H}_{2} \mathrm{O}}+10 Y_{\mathrm{H}_{2}}-Y_{\mathrm{O}_{2}} \ldots \ldots$

5 Laminar flame speeds (C1-C3) for the physical space solution (lines with symbols) and for the premixed flamelet model (red dots). . . . . . . . . . . . . . . . . . . . . . .

4 Comparison of premixed flamelet solutions (dot) to freely-propagating flame solutions (line) using a mixture-averaged diffusion model for hydrogen-air flames for different equivalence ratios (0.8-1.2, blue). The progress variable is defined as $Y_{c}=Y_{\mathrm{H}_{2} \mathrm{O}}-Y_{\mathrm{H}_{2}} \ldots \ldots \ldots \ldots \ldots \ldots$ Normalized budget of Eq. (21) over the normalized progress variable $c$ for a stoichiometric methaneair flame. The balance represents the sum of the three terms. . . . . . . . . . . . . . . . 